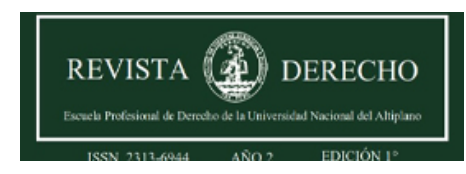

Revista de Derecho

ISSN: 2313-6944

ISSN: 2707-9651

revistaderecho@unap.edu.pe

Universidad Nacional del Altiplano

Perú

\title{
EL DELITO DE NOMBRAMIENTO ILEGAL PARA CARGO PÚBLICO EN EL DISTRITO JUDICIAL DE PUNO
}

Castro Mendoza, Karen Vanessa

EL DELITO DE NOMBRAMIENTO ILEGAL PARA CARGO PÚBLICO EN EL DISTRITO JUDICIAL DE PUNO

Revista de Derecho, vol. 5, núm. 2, 2020

Universidad Nacional del Altiplano, Perú

Disponible en: https://www.redalyc.org/articulo.oa?id=671870937009

DOl: https://doi.org/10.47712/rd.2020.v5i2.88

\section{(c) (1)}

Esta obra está bajo una Licencia Creative Commons Atribución 4.0 Internacional. 


\title{
EL DELITO DE NOMBRAMIENTO ILEGAL PARA CARGO PÚBLICO EN EL DISTRITO JUDICIAL DE PUNO
}

\author{
The illegal appointment crime for public office in the judicial district of Puno \\ Karen Vanessa Castro Mendoza \\ Corte Superior de Justicia de Puno, Perú \\ kvcm_2810@hotmail.com \\ DOI: https://doi.org/10.47712/rd.2020.v5i2.88 \\ Redalyc: https://www.redalyc.org/articulo.oa? \\ $\mathrm{id}=671870937009$
}

iD https://orcid.org/0000-0002-0018-030X

Recepción: 30 Septiembre 2020

Aprobación: 25 Octubre 2020

Publicación: 25 Octubre 2020

\section{Resumen:}

En el presente artículo, se abordarán cuestiones relacionadas a la interpretación y aplicación de los alcances del delito de nombramiento ilegal para cargo público, en el distrito judicial de Puno, en razón a que dicho ilícito, usualmente no es aplicado para el supuesto de una "designación” para cargo público; sino que se aplica específicamente para casos en los que se contrató a una persona bajo la modalidad de plazo indeterminado.

Asimismo, se tiene que dentro de los elementos objetivos de dicho injusto penal, también existe un gran debate, en relación a la aplicación del término "requisitos legales", creyéndose que dicho extremo solo abarcaría una norma con rango de ley, excluyendo a resoluciones administrativas que se dan dentro de una determinada institución pública, las mismas que fijan los requisitos a cumplir para un determinado perfil o puesto de trabajo, lo que en muchos casos, ha ocasionado que las personas procesadas bajo los alcances de dicho tipo penal, obtengan condenas, cuando en otros distritos judiciales, son absueltos por hechos similares, de ahí, se sostiene que la falta de claridad en el contenido del citado delito, conlleva a una mala interpretación y aplicación de esta figura por parte de los operadores de justicia, pues no existe un criterio uniforme al respecto.

Palabras clave: Funcionario público, contrato, nombramiento, designación, operadores de justicia.

\section{Abstract:}

This Article shall address issues relating to the interpretation and application of the scope of the offence of illegal appointment to public office, in the judicial district of Puno, on the reason that such wrongdoing is usually not applied in the event of a "designation" for public office; but applies specifically to cases in which a person was hired under the indeterminate time-limit modality.

It is also necessary that within the objective elements of such criminal unfairness, there is also a great debate, in relation to the application of the term "legal requirements", believing that that point would only cover a rule of law, excluding administrative decisions that take place within a given public institution, the same ones that lay down the requirements to be met for a particular profile or job, which in many cases has caused persons prosecuted under the scope of that criminal type to obtain convictions, when in other judicial districts, they are acquitted by similar facts, hence it is held that the lack of clarity in the content of that crime leads to a misinterpretation and application of this figure by the operators of justice, as there is no uniform criterion in this regard. KEYWORDS: Public official, contract, appointment, designation, operators of justice.

\section{INTRODUCCIÓN.}

En el presente artículo, se explicará en qué sentido se está realizando la interpretación de los elementos objetivos del artículo $381^{\circ}$ del Código Penal Peruano vigente, por parte de los operadores de justicia del Distrito Judicial de Puno. En ese entender, se determinará las situaciones en donde se observe consecuencias

\section{NotAS DE AUTOR}


jurídicas contrarias a las garantías procesales y a los derechos de los procesados, generado a consecuencia de la variada interpretación y aplicación del marco legal referido, específicamente al delito de nombramiento ilegal para cargo público.

\section{1.-Materiales y Métodos}

En el presente trabajo se tomará en cuenta el método Cualitativo, que permitirá realizar el análisis e interpretación de textos, doctrina, jurisprudencia y sentencias de primera y segunda instancia, relacionados al delito materia de interpretación. Se utilizará las fichas de observación y de anotación de resumen como instrumentos.

\section{El Delito de nombramiento ILEgal PARA CARgo PÚblico}

El tipo penal en mención, se encuentra regulado en el artículo $381^{\circ}$ del Código Penal vigente, el mismo que establece: "El funcionario público que hace un nombramiento para cargo público a persona en quien no concurren los requisitos legales, será reprimido con sesenta a ciento veinte días multa ...”.

\subsection{Antecedentes legales}

El citado ilícito penal no registra antecedentes en nuestra legislación penal, siendo que se ha encontrado como única fuente extranjera, el artículo $253^{\circ}$ del Código Penal de Argentina vigente, que establece: "Será reprimido con multa de SETECIENTOS CINCUENTA PESOS a DOCE MIL QUINIENTOS PESOS e inhabilitación especial de seis meses a dos años, el funcionario público que propusiere o nombrare para cargo público, a persona en quien no concurrieren los requisitos legales (...)”.

Es más, al revisar el Código Penal peruano del año 1924, se observa que el mismo no se encontraba regulado.

Conforme al jurista (Rojas, 2001), el nombramiento ilegal para cargo público en nuestro país se trata de una figura penal moderna de base estrictamente administrativa. Más aún pocas son las legislaciones penales que han elevado dicho supuesto administrativo a nivel de delito. El referido autor añade que estamos frente a un delito de resultado de naturaleza comisiva.

\section{INTERPRETACIÓN DEL DELITO DE NOMBRAMIENTO ILEGAL PARA CARGO PÚBLICO EN EL Distrito Judicial de Puno}

\subsection{Pronunciamiento del Primer Juzgado Penal Unipersonal de Puno.-}

De la revisión del Expediente $\mathrm{N}^{\circ}$ 00709-2017-44-2101-JR-PE-03, seguido en contra del Ex Alcalde Provincial de Puno - Iván Joel Flores Quispe, por la presunta comisión del delito de nombramiento ilegal para cargo público, se tiene que la Jueza del Primer Juzgado Penal Unipersonal de Puno, expidió una sentencia condenatoria en contra de dicho procesado, donde se le impuso setenta días multa, así como el pago por concepto de reparación civil, a favor de la parte agraviada y el pago de costas procesales al sentenciado; por habérsele encontrado responsable respecto a la imputación realizada por el representante del Ministerio Público, esto es:

Que, el denunciado Iván Joel Flores Quispe en su calidad de titular del pliego de la Municipalidad Provincial de Puno, habría designado en el cargo de Gerente General de la Municipalidad a Edgar Eloy Centeno Chavarría mediante Resolución de Alcaldía N 002-2015-MPP/A, de fecha 01 de Enero del 2015; siendo que Edgar Eloy Centeno Chavarría, no cumple con los requisitos mínimos requeridos por el Manual de Organización y Funciones (MOF) de la Municipalidad Provincial de Puno, esto es, que Edgar Eloy Centeno Chavarría no cuenta con título profesional y colegiatura entre otros para desempeñar el cargo. 
En ese sentido, entre los argumentos más importantes realizados por la Magistrada de primera instancia, se tienen los siguientes:

a) La conducta típica no es la del funcionario público que "nombra", sino la de aquel que "hace un nombramiento" para cargo público sobre persona en quien no concurren los requisitos legales.

b) Se ha designado a Edgar Eloy Centeno Chavarría en el cargo de Gerente General de la Municipalidad Provincial de Puno mediante Resolución de Alcaldía Nº 002-2015-MPP/A de fecha 01 de enero del 2015, sin que cumpla con los requisitos mínimos requeridos por el Manual de Organización y Funciones (MOF) de la Municipalidad Provincial de Puno, en razón de que no cuenta con título profesional y colegiatura para desempeñar el cargo.

c) Se expidió la Ordenanza Municipal Nº194-2008-CMPP, de fecha 18 de enero del 2008, mediante la cual el Alcalde de la Municipalidad Provincial de Puno aprueba el Cuadro de Asignación de Personal - CAP de dicha entidad municipal, así también se tiene el Decreto de Alcaldía No 001-2010/MPP/A, de fecha 26 de febrero del 2010, expedido por el Ing. Luis Butrón Castillo en su calidad de ex Alcalde de la Municipalidad Provincial de Puno, donde decreta aprobar el Manual de Organización y Funciones "MOF” de la Municipalidad Provincial de Puno, y es de verse de este que en relación al cargo estructural de Gerente Municipal se tiene establecido como requisito mínimo del cargo lo siguiente: 1 . Tener título profesional universitario, con colegiatura.

d) Del Currículum Vitae, que corresponde a la persona de Edgar Eloy Centeno Chavarría, se consigna en el rubro de formación universitaria que es graduado con estudios universitarios concluidos en la Facultad de Ingeniería Económica - UNA Puno Perú, en trámites para título profesional en proceso.

e) El acusado expidió el Decreto de Alcaldía No 002-2015/A-MPP, en fecha 27 de enero del 2015, mediante el cual modifica el Manual de Organización y Funciones - MOF de la Municipalidad Provincial de Puno, respecto a diferentes cargos y dentro de ellos el cargo de Gerente Municipal, estableciendo como requisitos mínimos del cargo lo siguiente: 1 . Tener título profesional universitario o amplia experiencia como responsable en la Gestión y Dirección de Corporaciones; entre otros.

Conforme a dichos argumentos, se logró advertir que el acusado Iván Joel Flores Quispe de manera muy conveniente modificó el Manual de Organización y Funciones de la entidad edil, respecto a los requisitos mínimos para asumir el cargo de Gerente General, hecho que era materia de discusión, pues con el supuesto añadido de "amplia experiencia", habría garantizado que el señor Edgar Centeno Chavarría desempeñe el cargo de Gerente Municipal de Puno.

En consecuencia, se ha señalado que no podría operar el error de tipo vencible ni invencible, a favor del acusado Iván Joel Flores Quispe, pues este conocía perfectamente los requisitos legales que debía reunir la persona que ocupará el cargo de Gerente Municipal como era que tenía que contar con título profesional, pero a pesar de ello, realizo una designación para dicho cargo, en una persona en la que no concurría los requisitos legales previstos en el MOF de la entidad municipal agraviada; por lo que, se le declaró autor del delito de nombramiento ilegal para cargo público.

\subsection{Decisión de la Sala Penal de Apelaciones de Puno. -}

La defensa técnica del acusado Iván Joel Flores Quispe, recurre en vía de apelación a la Sala Penal de Apelaciones de Puno, a efectos de que revisen la sentencia expedida en primera instancia, es así que, una vez llevado el trámite correspondiente y cerrado los debates, se programa fecha para la lectura de la sentencia de vista, la misma que se realizó en fecha 29 de noviembre del 2018. Siendo que dicho Colegiado expide la Sentencia de Vista N ${ }^{\circ}$ 174-2018 en MAYORÍA, existiendo un VOTO DISCORDANTE.

Considero que dicha resolución judicial da lugar a un debate jurídico en nuestra localidad, ello debido a que existe discrepancia en la interpretación realizada sobre dicho ilícito penal, donde una vez revisada la sentencia recurrida, se tiene que el Tribunal Superior, por MAYORÍA ha revocado la decisión de primera instancia, ABSOLVIENDO al citado ex funcionario público; esto en merito al análisis contrapuesto que se obtuvo de los elementos objetivos de dicho ilícito penal, esto es, en lo concerniente a "nombramiento" y 
"requisitos legales". Es así que de los argumentos invocados por dos de los Jueces Superiores que integran la Sala Penal de Apelaciones de Puno, para absolver al citado encausado, se tienen los siguientes:

a) El tipo penal referido hace alusión a "requisitos legales"; tales requisitos establecidos en el tipo penal en cuestión, en virtud del principio de legalidad y de reserva de la ley penal, del principio de igualdad y de aplicación general de la ley penal, solo puede y debe encontrarse en una norma con rango de ley o una norma de inferior jerarquía a la ley, pero de alcance general (nacional).

b) La Juez de Instancia ha validado como "requisito legal" el MOF de la Municipalidad Provincial de Puno aprobado por Resolución de Alcaldía No. 002-2015-MPP/A que establece la exigencia de título profesional con colegiatura para el cargo de confianza de Gerente Municipal. Nótese que el referido MOF se ha aprobado mediante un mero acto administrativo (resolución de Alcaldía). Dicha Resolución de Alcaldía no puede llenar el contenido típico del "requisito legal", en la medida que los alcances de la referida resolución solo tienen rigor y aplicación en la circunscripción territorial de la Comuna de la provincia de Puno y sus distritos. Darle contenido legal - penal generaría las siguientes ilogicidades de naturaleza penal, esto es que el delito de nombramiento ilegal de cargo público solo podría materializarse en dicha circunscripción territorial y no podría ser delito en otra provincia o provincias del territorio peruano en cuyas municipalidades su MOF no contenga la exigencia de título profesional para el cargo de Gerente Municipal. Esta conclusión interpretativa enfrenta y riñe el principio de aplicación general y el principio de igualdad de la ley penal en todo el territorio de la república (principio de aplicación territorial y extraterritorialidad contemplados en los artículos 1 al 5 del Código Penal); además que el MOF no tiene obligación normativa de publicarse en el diario oficial y al no haberse publicado se habría inobservado el artículo 109 de la Constitución; y que, en la eventualidad de que el MOF llenaría el contenido del tipo penal, podría darse el caso que una posterior modificación del mismo, en el que se establecería que ya no resulta necesaria la exigencia de título profesional colegiado para el cargo de Gerente Municipal, convertiría en atípico el hecho y como tal tendría que aplicarse retroactivamente la ley penal en aplicación del artículo 103 de la Constitución.

c) Finalmente puede darse la figura de que el referido tipo penal constituye una ley penal en blanco, que es aquella que se remite a una fuente jurídica de diferente calidad a la exigida por la Constitución Política, que puede ser otra ley penal, leyes de otros sectores de orden jurídico, normas reglamentarias de nivel inferior a la ley. La Ley penal en blanco, puede ser complementado por leyes de la misma materia (ley penal), por leyes extrapenales o por preceptos infra legales de carácter y alcance general en todo el territorio de la República. Las normas infra legales que no tengan alcance general (ordenanzas regionales, ordenanzas municipales), no pueden ser considerados para completar el tipo penal en blanco, en razón de que pueden generar, la punición en ciertas partes del territorio de la república y en otras partes son atípicos, lo que afecta el principio de igualdad ante la ley.

d) La Resolución de Alcaldía No. 002-2015-MPP/A que aprobó el MOF de la Municipalidad Provincial de Puno que establece la exigencia de título profesional con colegiatura para el cargo de confianza de Gerente Municipal. No se trata de una norma con rango de ley, ni siquiera es una norma infra legal de carácter general, ya que se trata de un mero acto administrativo y el incumplimiento de tal acto, solo acarrea consecuencias de carácter administrativo (sanción disciplinaria).

\subsection{Análisis del caso. -}

Es claro que la administración pública debe salvaguardar la eficacia e idoneidad profesional y personal de sus funcionarios, por ende, debe de garantizar la objetividad en la selección de su personal, esto con el cumplimiento de una mínima capacidad y aptitud para el ejercicio del cargo.

Conforme a lo señalado en los numerales que preceden, considero que el criterio asumido por dos de los magistrados de la Sala Penal de Apelaciones de Puno, es errado, en razón a que del caso concreto debió de verificarse la concurrencia de hasta dos elementos objetivos importantes que presenta este tipo penal: a) Hacer un nombramiento; b) El cargo público; y, c) Persona que no cuenta con los requisitos legales. 
Siendo que el Tribunal Superior, a efectos de absolver al encausado, debió de tomar en cuenta que el acto de nombrar a determinada persona, para ser legal, tiene que cumplir con las formalidades previstas no sólo en la Ley, sino que también estas pueden estar inmersas en un reglamento o resolución administrativa expedida por la institución pública donde se hace dicha designación o nombramiento.

Estimo que se debe de realizar la subsunción de los actos de designación o mera contratación a plazo fijo dentro del tipo penal de nombramiento ilegal para cargo público, pues dicha acción, también afecta o vulnera el bien jurídico consistente en la legalidad en la designación para cargos públicos en la administración pública, es decir, preservar la legalidad de los nombramientos de los funcionarios o servidores públicos, protegiéndola de irregularidades en el ingreso a la función pública. Es así que el derecho penal, al tipificar este delito pretende proteger, como señala el profesor (Cabrera, 2010) quien cita a Rojas Vargas, el óptimo desempeño de las tareas de la administración, que puede verse afectado cuando personas que no cuentan con las condiciones inherentes al cargo, asumen el puesto en contravención a la normatividad aplicable.

En pocas palabras, se debe de proteger el prestigio de la administración pública, ello con la búsqueda de que sus componentes humanos designados sean idóneos y de calidad, es decir, que no haya cuestionamientos sobre dichas cualidades, hecho que se realiza cuando dichos agentes públicos a designar cumplan con las condiciones inherentes al cargo que van a desempeñar, lo cual se encuentra establecido en sus respectivos reglamentos o resoluciones emitidas por la autoridad competente dentro de la institución pública donde se desenvolverán laboralmente.

Conforme a lo manifestado, es de considerar que el Tribunal Superior, se aparta de la jurisprudencia nacional, al no tomar en cuenta que el delito de nombramiento ilegal para cargo público, se consigna como la conducta típica no la del funcionario que nombra, sino la de aquel que "hace un nombramiento" para cargo público sobre persona en quien no concurren los requisitos legales, no describe un acto formal único, sino, que alude un procedimiento que se desarrolla en etapas, el cual se inicia cuando el funcionario elige a una determinada persona para que desempeñe un cargo público en particular, estableciendo, entre otras, las condiciones y horarios de trabajo, así como las funciones que desempeñará, y que finalizará cuando este lo disponga, siendo, por lo demás, su contratación un mero acto formal de carácter administrativo a cargo de la entidad correspondiente[1].

Es claro que el tipo penal materia de análisis, si bien hace referencia al elemento objetivo de "hacer un nombramiento", pero este también debe ser entendido como el acto de "hacer una designación", es decir, que el funcionario público hace un nombramiento cuando designa para un empleo o cargo público a una persona que necesariamente debe estar individualizada. Por lo que, es irrelevante determinar si el nombramiento es de carácter definitivo o provisional. En ambos supuestos es claro que se exige que el nombramiento para cargo público se realice sobre persona que no reúne los requisitos legales exigidos por ley o reglamento; aspecto que no ha sido debidamente valorado por el Tribunal Superior.

Asimismo, otro elemento objetivo fundamental en la tipicidad del delito analizado, lo constituye el hecho de que la persona a quien se nombra para desempeñar cargo público no reúne los requisitos legales previstos en la ley, reglamento o resolución administrativa previamente emitida por la institución pública de que se trate.

La expresión requisitos legales constituye una frase normativa que para el caso de nombramiento ilegal nos conduce a la normativa extrapenal, donde se prevé los requisitos legales que debe cumplir una persona para ser nombrada definitiva o provisionalmente en un cargo público (Reátegui, 2016).

Dicho extremo es sumamente importante, pues cuando se hace alusión a "requisitos legales", estos deben ser entendidos como aquellas características relevantes con las que debe contar la persona sobre la cual se hará un nombramiento o designación, las mismas que las encontraremos en la ley, reglamento o cualquier otra resolución emitida por la entidad pública que corresponda.

Naturalmente ello supone observar la estricta confluencia de todos los requisitos legales exigidos en las calidades del aspirante. El incumplimiento doloso de estos requisitos es lo que torna penalmente relevante el supuesto de hecho. 
En el mismo sentido se ha pronunciado el jurista (Reátegui, 2015) al establecer que en este tipo penal la conducta típica se configura cuando: “... El nombramiento para ser legal tiene que cumplir con las formalidades previstas en la ley, reglamento o resolución administrativa de la institución pública donde se hace la designación".

Siendo que, omitir observar debidamente los requisitos legales, por parte del funcionario, actúa aquí como presupuesto de la conducta de nombramiento ilegal de naturaleza activa; es decir, aquí el agente debe conocer perfectamente los requisitos exigidos por ley, reglamento o resolución administrativa que debe reunir la persona a la que nombró o designó para que ocupe determinado cargo público, siendo que en caso contrario, el agente incurriría en un error de tipo vencible o invencible según corresponda.

En sentido opuesto a lo que señala la Sala Penal de Apelaciones de Puno, estimo que si bien es cierto la norma penal es de aplicación general a todo el país; ello no impide que conforme a las normas extrapenales de una determinada ciudad, como es la ciudad de Puno, pueda darse el delito de nombramiento ilegal para cargo público, por cuanto la conducta establecida en la norma penal, vale decir, en el artículo $381^{\circ}$ del Código Penal, está regulada en función a la norma, reglamento o resolución vigente, en la cual se señalan los requisitos para ejercer o aspirar a un determinado cargo, en el caso concreto, las mismas estaban fijadas en el MOF de la Municipalidad Provincial de Puno, las cuales fueron fijadas conforme a las exigencias, requerimientos o realidad del lugar donde se emitieron; de tal manera que si dichos "requisitos" no son observados por parte del funcionario público que realiza el nombramiento (sujeto activo), tal situación conlleva a incurrir en el delito materia de análisis; pues, lo que está en juego es el cumplimiento de los requisitos establecidos en una "norma extrapenal”, que es de libre determinación de la autoridad que la emite, empero que debe ser cumplida, en tanto no haya sido derogada, modificada o dejada sin efecto, por lo que, en ese sentido nada impide que por la divergencia que existe en dichas normas administrativas, en algunos lugares, se pueda incurrir en dicho delito y en otros no.

Se puede advertir que el tipo penal analizado no hace alusión a normas con rango de ley, sino a la inconcurrencia de requisitos legales, es decir, a los requisitos exigidos por ley, por lo que, considero que la interpretación de dicho término se entiende referido a cualquier norma extrapenal; extremo que así también ha sido interpretado y precisado por el jurista (Salinas, 2016): "Otro elemento objetivo fundamental en la tipicidad del delito lo constituye el hecho que la persona a quien se nombre para desempeñar cargo público no reúne los requisitos legales previstos en la ley, reglamento o resolución administrativa previamente emitida por la institución pública de que se trate". Cuya interpretación es la correcta, por cuanto lo que se protege con la tipificación de dicho ilícito, es la preservación de la legalidad de los nombramientos de los funcionarios o servidores públicos.

En este escenario, analizado el pronunciamiento tanto de primera como de segunda instancia, considero que el Tribunal Superior en su mayoría, realizó un indebido análisis de dos de los elementos objetivos del delito tipificado en el primer párrafo del artículo $381^{\circ}$ del Código Penal; tal como lo he mencionado, apartándose de la jurisprudencia y doctrina nacional a efectos de sustentar su decisión; en consecuencia, si habrían tomado en cuenta éstas, no les quedaba más que confirmar la responsabilidad penal del encausado; por ende, no se hubiera dejado sin sanción penal una conducta que se encontraba debidamente tipificada, como ocurrió en el caso analizado.

\section{CONCLUSIONES}

El correcto funcionamiento de todas las instancias de la administración pública, no solo tiene que ver con el apego estricto al principio de legalidad de los funcionarios y servidores públicos, sino también de que aquellos cuenten con las condiciones y calificaciones adecuadas, para que la gestión pública puede prestar a la ciudadanía un servicio de calidad. Es así, que las plazas o puestos en la administración pública se encuentran 
legalmente reguladas, mediando la elaboración de un perfil técnico profesional, donde solo si el postulante cumple con los requisitos exigidos es que puede acceder al cargo o puesto.

Sobre el delito de nombramiento ilegal para cargo público, corresponde recoger en el mismo el verbo rector de "designar"; aspecto que nuestros operadores de justicia deben de considerar, esto con la finalidad de no incurrir en interpretaciones contradictorias y absolver o sancionar injustamente a los encausados; pues se diría que solo aquel que nombra o contrata a una persona a plazo indeterminado podría ser autor del delito. Lo mismo ocurre con el elemento objetivo de "requisitos legales", el cual debe ser entendido como las formalidades previstas en la ley, reglamento o resolución administrativa de determinada institución pública donde se pretende hacer la designación de una persona para que ocupe un puesto en la administración pública.

\section{BIBLIOGRAFÍA.}

1. PEÑA CABRERA FREYRE, Alonso Raúl. "Derecho Penal Parte Especial" Tomo V. Primera Edición. Editorial IDEMSA. Lima Perú, 2010.

2. REATEGUI SÁNCHEZ James; "Delitos con la administración pública en el Código Penal”; Jurista Editores, Lima Perú, 2015.

3. REÁTEGUI SÁNCHEZ, James. "Tratado de Derecho Penal - Parte Especial”Volumen 3. Primera Edición, Legales Ediciones, Lima Perú, 2016

4. ROJAS VARGAS, Fidel. "Delitos Contra La Administración Pública”. Segunda Edición. Editorial GRIJLEY. Lima Perú, 2001.

5. SALINAS SICCHA, Ramiro. "Delitos Contra La Administración Pública”. Segunda Edición. Editorial GRIJLEY. Lima Perú, 2016.

\section{Notas}

[1] Resolución Suprema del 04 de mayo del 2010, en el Recurso de Apelación del Exp. № 07-2007 de la Sala Penal Especial de la Corte Suprema integrado por los jueces supremos Prado Saldarriaga (ponente), Príncipe Trujillo y Santa María Morillo. 